\title{
Comparative study between fixed and flexible GnRH antagonist protocol versus GnRH agonist long protocol in polycystic ovarian disease patients treated with IVF
}

\author{
Hany Maged Abd-Elaal ${ }^{a}$, Farid Ahmed Kassab ${ }^{a}$, Osama Deif ${ }^{a}$, Ismael Abd-Elazeem Mira ${ }^{a}$, \\ Mohamed Mohamed Mohamed Essawy ${ }^{b}$ \\ ${ }^{a}$ Obstetrics and Gynecology department, Faculty of Medicine, Al-Azhar University, ${ }^{\mathrm{b}}$ Obstetrics and \\ Gynecology Department, Beni-Suef General Hospital, Ministry of Health, Egypt.
}

\begin{abstract}
:
The objective of the present study was to compare and evaluate the effectiveness and safety of GnRH agonist long protocol compared with the GnRH antagonist (fixed and flexible) protocols in polycystic ovary syndrome (PCOS) patients undergoing in vitro fertilization (IVF)/ intracytoplasmic sperm injection (ICSI) cycles. This study was carried out on 60 patients with polycystic ovarian disease undergoing ICSI, their age ranged from (20 to 40 years). The cases were selected from The International Center for Population Study and Research (ICPS), Al-Azhar University. Eligible patients who accepted to take part in the study were randomized into 3 study group: Group A: 20 patients were included in agonist protocol, Group B: 20 patients were included in fixed antagonist protocol and Group C: 20 patients were included in flexible antagonist protocol. The results obtained from this study indicated no statistically significant differences regarding the pregnancy rates or regarding the developing of ovarian hyperstimulation syndrome (OHSS) complication in the studied population; so larger studies with larger sample size and longer duration are needed to clarify the roles of different IVF protocols.
\end{abstract}

Keywords:

Gonadotropin-releasing hormone agonist, gonadotropin-releasing hormone antagonist, in vitro fertilization, polycystic ovarian syndrome

\section{Introduction:}

Polycystic ovary syndrome (PCOS) is a common endocrinal disorder affecting $6.6-8 \%$ of women in childbearing period ${ }^{(1)}$. It is associated with $75 \%$ of the causes of anovulatory infertility ${ }^{(2)}$.

The polycystic ovary syndrome (PCOS) is a heterogeneous collection of signs and symptoms that, gathered together, form a spectrum of a disorder with a mild presentation in some and in others a severe disturbance of reproductive, endocrine and metabolic function. The definition of the syndrome has been much debated. Key features include menstrual cycle disturbance, hyperandrogenism and obesity.There are many extra-ovarian aspects to the pathophysiology of PCOS, yet ovarian dysfunction is central. At a joint consensus meeting of the American Society for Reproductive Medicine and the European Society of Human Reproduction and Embryology (ASRM/ESHRE) a refined definition of the PCOS was agreed,namely the presence of two out of the following three criteria: oligo- and/or anovulation, hyperandrogenism (clinical and/or biochemical), polycystic ovaries with the exclusion of other etiologies. The morphology of the polycystic ovary has been redefined as an ovary with 12 or more follicles measuring 2-9 $\mathrm{mm}$ in diameter and/or increased ovarian volume $(>10 \mathrm{~cm} 3)^{(3)}$.

The pathophysiology of PCOS is likely to be multifactorial and polygenic. There is a significant body of evidence suggesting that excess ovarian androgen production is central in the pathogenesis of PCOS ${ }^{(4)}$.

One of the main problems occurring in patients with PCOS undergoing IVF / ICSI is developing ovarian hyperstimulation syndrome (OHSS); a serious iatrogenic complication of ovarian stimulation induced and triggered by exogenous and/or endogenous HCG which varies from mild to severe and critical forms ${ }^{(5)}$.

The long GnRH agonist protocol has been used for pituitary desensitization in patients with PCOS undergoing IVF / ICSI with the benefit of significant reduction in the occurring of premature LH surges and the frequency of cycle cancellation ${ }^{(6)}$.

GnRH antagonist down-regulation protocol in IVF / ICSI has gained much popularity over the last few years ${ }^{(7)}$. It acts by competitive inhibition of GnRH receptors in pituitary, and produce an immediate and rapid reduction in $\mathrm{LH}$ and FSH levels without GnRH receptor 
desensitization as well as flare-up effect. Previous studies have shown that GnRH antagonist protocols decrease the incidence of OHSS as well as the amount of gonadotropins used and the duration of stimulation as compared with GnRH agonist protocols in the general population ${ }^{(8)}$.

In the last years, there was more interest in using GnRH antagonist protocol in patients with PCOS treated with IVF with the aim of reducing the incidence of OHSS in this vulnerable group of patients. Recent studies showed that GnRH antagonist protocol to be as effective as the GnRH agonist LP in PCOS patients with lower rates of OHSS ${ }^{(\boldsymbol{9})}$.

\section{Patients and Methods}

This study was carried out on 60 patients with polycystic ovarian disease undergoing ICSI. The cases was selected from The International Center for Population Study and Research (ICPS), AlAzhar University. The study was approved by the Ethics Board of Al-Azhar University.

\section{Inclusion criteria}

- Age group between 20-40 years.

- Normal prolactin and thyroid function tests.

- Normal cardiac, hepatic and renal functions.

- Normal spontaneous onset of puberty and normal sexual development.

- Normogonadotrophic females.

- Day 2 FSH level below 10 IU/L.

- PCOS patients (fulfilling Rotterdam criteria of PCOS $)^{(3)}$.

- Body mass index (BMI) $<35 \mathrm{~kg} / \mathrm{m} 2$.

\section{Exclusion criteria}

Poor response in previous intracytoplasmic sperm injection (ICSI) cycles.

- History of previous ovarian surgery.

- Uterine factor infertility.

- Severe male factor infertility.

- Patients with endometriosis or ovarian cyst.

- Thyroid and prolactin disorders.

- Anatomical abnormality in uterus or cervix or hydrosalpinx.

- Disorders in cardiac, hepatic and renal functions.

\section{Randomization:}

Eligible patients who accepted to take part in the study were randomized into 3 study group Group A: 20 patients were included in agonist protocol.

Group B: 20 patients were included in fixed antagonist protocol.

Group C: 20 patients were included in flexible antagonist protocol.

\section{Ovarian stimulation}

All patients received oral contraceptive pills starting on day 4 of spontaneous menses of the cycle prior to the treatment cycle for 21 days pretreatment in the cycle preceding ovarian stimulation.

Ovarian stimulation was commenced on day 2 of spontaneous or progesterone withdrawal bleeding. The starting dose was adjusted according to patient's age, antral follicle count (AFC) and prior response to gonadotropin stimulation as per unit protocol. We used step up protocol of gonadotropin stimulation and the dose was adjusted every 3-4 days according to ovarian response. The gonadotropin preparations used were highly purified FSH (Fostimone (IBSA, Switzerland) and highly purified hMG (Merional, IBSA, Switzerland).

In Group A: GnRH agonist (long protocol) group, GnRH agonist, lucrine (Abbott Cedex, Istanbul, Turkey) $0.1 \mathrm{mg}$ / day was started on day 21 of the pre-treatment cycle. When pituitary desensitization was achieved, ovarian stimulation was started and the GnRH agonist was decreased to $0.05 \mathrm{mg}$ / day till day of HCG. Down regulation was confirmed by biochemical markers ( $\mathrm{LH}<5 \mathrm{IU} / \mathrm{ml}$, E2 $<50 \mathrm{pg} / \mathrm{ml}$ and progesterone $<1 \mathrm{ng} / \mathrm{ml}$ ) and transvaginal ultrasound (TVS) assessment of endometrial thickness (ET) and ovarian status (ET $<3 \mathrm{~mm}$, no ovarian cyst $>2 \mathrm{~cm}$ ).

In Group B: (fixed antagonist protocol), we started the gonadotrophin on cycle day 2 when ovarian suppression is assured. Then GnRH antagonist $0.25 \mathrm{mg}$ cetrorelix; cetrotide (Serono) was given daily on stimulation day 6 of menstrual cycle and was continued till the day of hCG. 
In Group C (flexible antagonist protocol ), Ovarian stimulation was commenced on day 2 of the cycle, daily intramuscular injection of gonadotrophin was started, $0.25 \mathrm{mg}$ of $\mathrm{GnRH}$ antagonist (cetrorelix acetate, Cetrotide; Merck Serono SA, Switzerland) was started when a leading follicle reached $14 \mathrm{~mm}$ and continued till the day of HCG.

\section{Ovarian triggering}

Ovarian follicular response was monitored with transvaginal ultrasound. Ultrasound scanning was started on stimulation day 7 then every other day. HCG injection was given (Choriomone 10,000 IU im, Choriomone, IBSA, Switzerland) when at least 3 follicles greater than $16 \mathrm{~mm}$ in diameter were detected on transvaginal ultrasound scan with the leading follicle reached $18-20 \mathrm{~mm}$ in diameter.

Oocyte retrieval was performed under anesthesia 36 hours after HCG administration. Fertilization was performed by standard IVF or ICSI. Cleavage stage embryo transfer (ET) was performed on day 2 or day 3 .

Embryo transfer was performed under abdominal ultrasound guide for proper embryo placement to the mid-uterine cavity. Two to five grade A or B embryos were transferred as per unit protocol. Embryo transfer was performed with a Wallace catheter (Smith Medical International Ltd, Hythe, Kent, UK).

Progesterone support of luteal phase was commenced on the day of ET with $800 \mathrm{mg}$ micronized progesterone vaginally till 12 weeks of pregnancy.

A serum HCG pregnancy test was performed 14 days after ET. Clinical pregnancies were confirmed by at least one ultrasonographically confirmed viable fetus within the uterus 4 weeks after ET.

\section{Statistical methodology:}

Statistical analysis was performed using the Statistical Package for Social Science (SPSS Inc, Chicago) version 17 for Microsoft Windows. Data were described in terms of mean \pm SD (standard deviation) for continuous variables and frequencies (number of cases) and percentages for categorical data. A one-way analysis of variance (ANOVA) was used when comparing between more than two means, kruskall Wallis test for multiple group comparisons in nonparametric data and Chi square test was used to compare categorical data.

$P$ value $<0.05 \%$ was considered significant.

\section{Results:}

This study was carried out on 60 patients with polycystic ovarian disease undergoing ICSI, they then divided into three protocols; 20 patients for each one; (20 patient for long agonist protocol, 20 patients for fixed antagonist protocol and 20 patients for flexible antagonist protocol).

Table (1): Baseline characteristics of the studied population; $(\mathrm{N}=60)$ :

\begin{tabular}{l|l|l|l|l}
\hline Characteristics & Group (A) & Group (B) & Group (C) & \\
& $\mathrm{N}=20$ & $\mathrm{~N}=20$ & $\mathrm{~N}=20$ & P value \\
\hline Age $($ years) & $32.55 \pm 3.634$ & $31.75 \pm 3.447$ & $30.75 \pm 3.972$ & $\mathrm{NS}$ \\
\hline BMI $(\mathrm{kg} / \mathrm{m} 2)$ & $24.85 \pm 2.183$ & $24.25 \pm 1.372$ & $24.75 \pm 1.970$ & $\mathrm{NS}$ \\
\hline FSH $(\mathrm{m} \mathrm{IU} / \mathrm{ml})$ & $6.05 \pm 0.887$ & $6.45 \pm 0.945$ & $5.90 \pm 0.852$ & $\mathrm{NS}$ \\
\hline E2 $(\mathrm{pg} / \mathrm{ml})$ & $42.20 \pm 6.818$ & $42.00 \pm 1.522$ & $43.80 \pm 6.118$ & $\mathrm{NS}$ \\
\hline
\end{tabular}

$P$ value $\leq .05$ significant $(S), P$ value $\geq .05$ Non significant (NS

Table (1) summarized the baseline characteristics of patients enrolled in the three protocol groups.

There was no significant difference in mean age, BMI, day 2 FSH, and E2 level

Table (2): Causes of infertility in the studied population; $(\mathrm{N}=60)$ :

\begin{tabular}{|c|c|c|c|c|}
\hline \multirow[b]{2}{*}{ Causes of infertility } & \multicolumn{3}{|l|}{$\mathrm{N}(\%)$} & \\
\hline & Group (A) & Group (B) & Group (C) & $P$ value \\
\hline
\end{tabular}




\begin{tabular}{ll|l|ll}
\hline & $\mathrm{N}=20$ & $\mathrm{~N}=20$ & $\mathrm{~N}=20$ & \\
\hline Tubal & $8(40 \%)$ & $7(35 \%)$ & $9(45 \%)$ & $\mathrm{NS}$ \\
\hline Unexplained & $6(30 \%)$ & $6(30 \%)$ & $5(25 \%)$ & NS \\
\hline Male & $4(20 \%)$ & $5(25 \%)$ & $4(20 \%)$ & NS \\
\hline Male and female & $2(10 \%)$ & $2(10 \%)$ & $2(10 \%)$ & NS \\
\hline
\end{tabular}

$P$ value $\leq .05$ significant $(S) . P$ value $\geq .05$ Non significant $(N S)$

Table (2) illustrated the causes of infertility among the studied groups; the different causes of infertility were nearly equally distributed among the studied groups with no statistically significant difference. Tubal factor was highest in group (C) while unexplained infertility was lowest in the same group. Male infertility was highest among group (B) and mixed (male and female) infertility was highest in group (C); however all differences were not significant statistically ( $p$-values $>0.05)$.

Table (3): Hormonal data of the studied 3 groups; $(\mathrm{N}=60)$ :

\begin{tabular}{l|l|l|l|l}
\hline \multirow{4}{*}{ Data } & \multicolumn{2}{l|}{ Mean \pm SD } & \\
\cline { 2 - 5 } & $\begin{array}{l}\text { Group }(\mathrm{A}) \\
\mathrm{N}=20\end{array}$ & $\begin{array}{l}\text { Group }(\mathrm{B}) \\
\mathrm{N}=20\end{array}$ & $\begin{array}{l}\text { Group }(\mathrm{C}) \\
\mathrm{N}=20\end{array}$ & \multirow{2}{*}{ P value } \\
\hline Days of HMG & $13.55 \pm 1.356$ & $9.85 \pm 1.424$ & $8.15 \pm .875$ & $\mathrm{~S}$ \\
\hline Doses of HMG & $2369.50 \pm 738.580$ & $1690 \pm 197.084$ & $1502.50 \pm 788.482$ & $\mathrm{NS}$ \\
\hline Endometrial thickness & $13.05 \pm 1.395$ & $9.90 \pm 1.832$ & $11.05 \pm 1.468$ & $\mathrm{~S}$ \\
\hline E2 level at HCG & $2727.50 \pm 363.273$ & $2965.00 \pm 318.343$ & $1450.00 \pm 280.507$ & $\mathrm{~S}$ \\
\hline
\end{tabular}

As summarized in table (3); the days of HMG was significantly highest among the group (A) with a mean of $13.55 \pm 1.356$ as compared with group (B) and group (C) where the mean was $9.85 \pm 1.424$ and $8.15 \pm .875$ days for groups (B) and (C) respectively with p-value $<0.05$.

Regarding the dose of HMG; no statistically significant difference was detected between the three groups.

The endometrial thickness was significantly highest among the group (A) with a mean of $13.05 \pm 1.395$ $(\mathrm{mm})$ as compared with group $(\mathrm{B})$ and group $(\mathrm{C})$ where the mean was $9.90 \pm 1.832$ and $11.05 \pm 1.468$ $(\mathrm{mm})$ for groups $(\mathrm{B})$ and $(\mathrm{C})$ respectively with p-value $<0.05$.

E2 level at HCG was significantly lowest among the group (C) with a mean of $1450.00 \pm 280.507$ as compared with group (A) and group (B) where the mean was $2727.50 \pm 363.273$ and $2965.00 \pm 318.343$ for groups (A) and (B) respectively with p-value $<0.05$.

Table (4): Clinical data of the studied 3 groups; $(\mathrm{N}=60)$ :

\begin{tabular}{|c|c|c|c|c|}
\hline \multirow[b]{2}{*}{ Data } & \multicolumn{3}{|l|}{ Mean \pm SD } & \multirow[b]{2}{*}{$P$ value } \\
\hline & $\begin{array}{l}\text { Group (A) } \\
\mathrm{N}=20\end{array}$ & $\begin{array}{l}\text { Group (B) } \\
\mathrm{N}=20\end{array}$ & $\begin{array}{l}\text { Group (C) } \\
\mathrm{N}=20\end{array}$ & \\
\hline N. of oocytes retrieved & $12.55 \pm 1.317$ & $11.35 \pm 1.899$ & $10.55 \pm 1.395$ & NS \\
\hline N. of MII oocyte & $8.30 \pm 1.261$ & $7.30 \pm 1.418$ & $5.90 \pm .912$ & $S$ \\
\hline N. of MI oocyte & $2.90 \pm 788$ & $2.15 \pm .875$ & $2.00 \pm .858$ & NS \\
\hline N. of degenerate oocyte & $2.30 \pm 0.865$ & $2.90 \pm .788$ & $2.05 \pm .826$ & NS \\
\hline
\end{tabular}

As regard the number of oocytes retrieved; no statistically significant difference was detected between the three groups.

Number of MII oocyte was significantly lowest among the group (C) with a mean of $5.90 \pm .912$ as compared with group (A) and group (B) where the mean was $8.30 \pm 1.261$ and $7.30 \pm 1.418$ for groups (A) and (B) respectively with p-value $<0.05$. However; no statistically significant difference was detected between the three groups regarding the number of MI oocyte. The same finding was observed 
regarding the number of degenerate oocyte where no statistically significant difference was detected between the three groups.

Table (5): Number of embryos between studied 3 groups; $(\mathrm{N}=60)$ :

\begin{tabular}{l|l|l|l|l}
\hline \multirow{4}{*}{ Data } & \multicolumn{2}{l|}{ Mean \pm SD } & \multirow{2}{*}{ Group (C) } \\
\cline { 2 - 5 } & Group (A) & $\begin{array}{l}\text { Group (B) } \\
\mathrm{N}=20\end{array}$ & 20 & P value \\
\hline Number of embryos & $7.15 \pm 1.182$ & $6.20 \pm 1.105$ & $4.80 \pm 1.105$ & $\mathrm{~S}$ \\
\hline Number of ET & $2.85 \pm .813$ & $2.90 \pm .788$ & $2.80 \pm .834$ & $\mathrm{NS}$ \\
\hline
\end{tabular}

As regard the number of embryos; the number was significantly lowest among the group (C) with a mean of $4.80 \pm 1.105$ embryos as compared with group (A) and group (B) where the mean was 7.15 \pm 1.182 and $6.20 \pm 1.105$ for groups (A) and (B) respectively with p-value $<0.05$.

Regarding the number of ET; no statistically significant difference was detected between the three groups.

Table (6): Pregnancy rates of ICSI cycles between 3 groups; $(\mathrm{N}=60)$ :

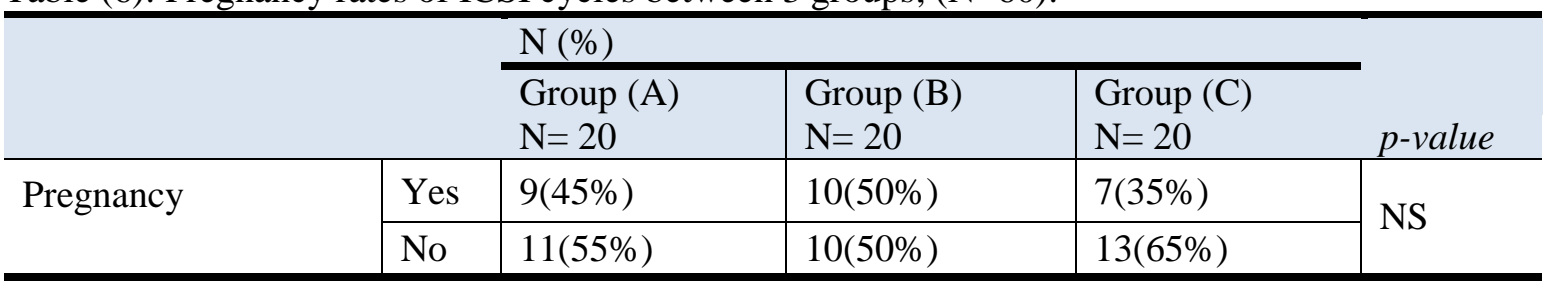

As demonstrated in table (6); pregnancy was confirmed in 9 cases (45\%) of group (A), 10 cases (50\%) of group (B) and 7 cases (35\%) of group (C) with no statistically significant difference between the studied 3 groups p-value $>0.05$

Table (7): Cycle cancellation rate among the 3 groups; $(\mathrm{N}=60)$

\begin{tabular}{|c|c|c|c|c|c|}
\hline & & \multicolumn{3}{|l|}{$\mathrm{N}(\%)$} & \multirow[b]{2}{*}{$p$-value } \\
\hline & & $\begin{array}{l}\text { Group (A) } \\
\mathrm{N}=20\end{array}$ & $\begin{array}{l}\text { Group (B) } \\
\mathrm{N}=20\end{array}$ & $\begin{array}{l}\text { Group (C) } \\
\mathrm{N}=20\end{array}$ & \\
\hline \multirow{2}{*}{ Cancellation } & Cancelled & $2(10 \%)$ & $2(10 \%)$ & $1(5 \%)$ & \multirow{2}{*}{ NS } \\
\hline & Not cancelled & $18(90 \%)$ & $18(90 \%)$ & $19(95 \%)$ & \\
\hline
\end{tabular}

As mentioned in table (7); protocol cancelation was done for 5 cases; 2 cases (10\%) of group (A), 2 cases $(10 \%)$ of group (B) and only one case (5\%) of group (C) with no statistically significant difference between the studied 3 groups ( $\mathrm{p}$-value $>0.05$ ).

Table (8): Causes of Cycle cancellation among the 3 groups; $(\mathrm{N}=5)$ :

\begin{tabular}{|c|c|c|c|c|c|}
\hline & & \multicolumn{3}{|l|}{$\mathrm{N}(\%)$} & \multirow[b]{2}{*}{ p-value } \\
\hline & & $\begin{array}{l}\text { Group (A) } \\
\mathrm{N}=2\end{array}$ & $\begin{array}{l}\text { Group (B) } \\
\mathrm{N}=2\end{array}$ & $\begin{array}{l}\text { Group }(\mathrm{C}) \\
\mathrm{N}=1\end{array}$ & \\
\hline \multirow{2}{*}{ Causes } & Poor responder & $1(5 \%)$ & $1(5 \%)$ & $1(5 \%)$ & \multirow{2}{*}{ NS } \\
\hline & Negative fertilization & $1(5 \%)$ & $1(5 \%)$ & $0(0 \%)$ & \\
\hline
\end{tabular}

An regarding the causes of cancellation in the 5 cancelled cases; as mentioned in table (8)

Three cases were cancelled due to poor response (one case in each group of the three studied groups) and 2 cases were cancelled due to negative fertilization [ 1 case from group (A) and the other case from group (B)] with no statistically significant difference between the studied 3 groups ( $\mathrm{p}$-value $>0.05$ ). 
Hany Abd-Elaal et al.

Table (9): Moderate ovarian hyperstimulation syndrome (OHSS) rates in the studied 3 groups; $(\mathrm{N}=60)$ :

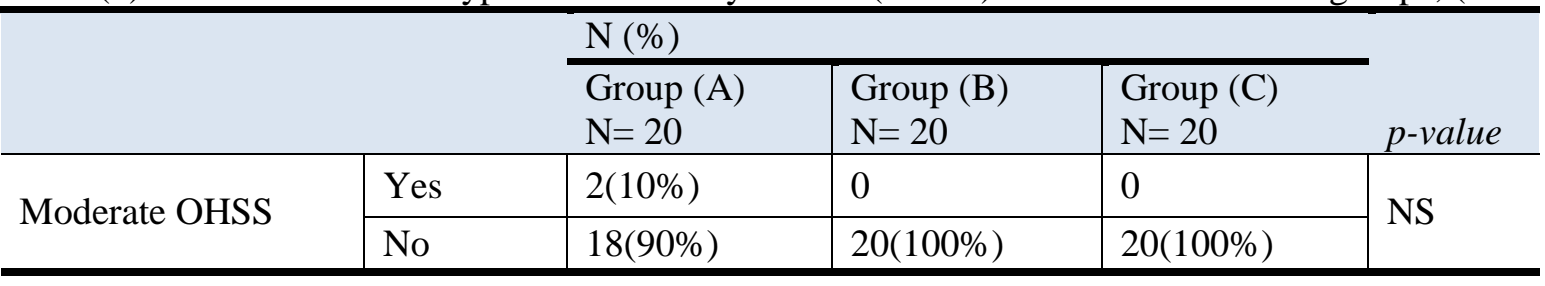

As mentioned above; 2 patients (10\%) of group (A) developed moderate OHSS, but none of the patients in group (B) and group (C) had this complication with no statistically significant difference between the studied 3 groups p-value $>0.05$

\section{Discussion}

The present study aimed to compare between $\mathrm{GnRH}$ agonist long protocol and $\mathrm{GnRH}$ antagonist protocols (fixed and flexible) in cases of polycystic ovarian diseases (PCO) treated by in vitro fertilization (IVF). The study was carried out on 60 patients with PCOS undergoing ICSI, 20 patients for each protocol where the cases were selected from The International Center for Population Study and Research (ICPS), AlAzhar University.

In our study; the patients' age ranged from 20 40 years old with no statistically significant differences between the three studied groups. By including only patients with BMI $<35$, we excluded the possibility of the negative effect of high BMI on LH surge ${ }^{(\mathbf{1 0})}$, the three studied groups had no significant differences regarding BMI. The distribution of causes of infertility in our studied population was similar among the three studied groups with no statistically significant difference between them.

Regarding the duration of administration of HMG to attain ovarian stimulation for IVF; there was a significant difference in the duration length were it was longest among the agonist protocol (Group A) with a mean duration of (13.55 \pm 1.356 days), while in the fixed antagonist protocol and the flexible antagonist protocol, the duration was shorter. Our finding was matched with many other studies as the reported by Bahçeci et al. where they reported a significantly lower duration in antagonist group (11). However; no difference in days of stimulation between two groups were reported by Kaur $\boldsymbol{e t}$ al., in their published prospective controlled study comparing long agonist protocol with flexible antagonist protocol ${ }^{\mathbf{( 1 2 )}}$.

Regarding the total gonadotrophin units required for stimulation; our results revealed no statistically significant difference in the required dose between the three studied groups which was opposite to the reported in a similar study in this regard where total dose of gonadotrophins was significantly lower in antagonist group $^{(\mathbf{1 2})}$; however Ashrafi and his colleges reported no statistically significant decrease in amount of total gonadotrophin units required although it was very near to significant level ${ }^{(13)}$. The endometrial thickness was significantly highest among agonist protocol (Group A) with a mean thickness of $(13.05 \pm 1.395 \mathrm{~mm})$ followed by flexible antagonist protocol. Endometrial thickness was significantly smallest among females in fixed antagonist protocol as compared to other two groups with a mean of $(9.90$ \pm 1.832 ). Our results were similar to the results of Huang et al. ${ }^{(14)}$, whose study included patients undergoing IVF and embryo transfer ${ }^{(14)}$. We have not been able to prove that the reduced endometrial thickness has any impact on the CPR. The fertilization rate showed no differences between the groups.

According to serum E2 level on day of hCG injection, females in fixed antagonist protocol group had significantly lower E2 level as compared to other two groups with a mean of $(1450.00 \pm 280.507)$. On the contrary, Ashrafi and his colleges reported no statistically significant difference of E2 level between the two groups in their study ${ }^{(\mathbf{1 3})}$.

The total number of retrieved oocytes were higher in agonist protocol (Group A) but without statistically significant difference between the studied groups. Although it was not statistically significant difference but it is opposite to the reported in many other studies that reported decrease in number of oocytes and other embryological data in the antagonist group than the agonist group ${ }^{(15-17)}$, but most of these studies was done on general populations (PCO and non PCO patients). 
The pregnancy rates in the present study was not significantly different between the studied three groups $(45 \%, 50 \%$ and $35 \%)$ in the agonist, fixed antagonist and flexible antagonist protocols respectively. Our findings were different than the reported in many other studies where; Orvieto and his collegesfound significant higher pregnancy rate in long agonist protocol compared with the GnRH antagonist protocol (36\% vs. $19 \%$ ) while two other similar studies showed no difference in pregnancy rate between two regimens ${ }^{(\mathbf{1 8 - 2 0 )}}$.

Lainas and his colleges compared flexible $\mathrm{GnRH}$ antagonist protocol with long agonist protocol in PCOS patients and found that there was no difference in ongoing pregnancy rate. They concluded that, antagonist protocol should be the treatment of choice in PCOS patients ${ }^{(21)}$. More other studies also reflected the same view (22-23).

In this present study; protocol cancelation was done for 5 cases only; 2 cases of group (A), 2 cases of group (B) and only one case of group (C) with no statistically significant difference between the studied 3 groups. This finding was comparable with many other studies in this regard as the reported in a similar study to compare different protocols of GnRH where the cancellation rate was higher than the reported in our study but did not significantly differ between the studied groups ${ }^{(24)}$.

Moderate ovarian hyperstimulation syndrome (OHSS) in the present study developed only in 2 cases from the studied population and both cases belonged to the GnRH agonist protocol group; but without statistically significant difference between the studied groups. This statistically insignificant difference may be because of the number of patients included in the study. A similar study in an Indian experience in 2012 of $\mathrm{GnRH}$ long agonist versus flexible $\mathrm{GnRH}$ antagonist protocol in PCOS reported 16 cases with OHSS in the agonist protocol which was significantly higher than the 2 cases reported in the antagonist protocol ${ }^{(\mathbf{1 2})}$.

Pundir et al. ${ }^{(25)}$ in a meta-analysis, which included 9RCTS with 966 women, tried to find whether GnRH antagonist protocol reduces the risk of OHSS in PCOS patients. There was no difference in severe OHSS rate but when moderate and severe OHSS cases were pooled, there was significant lower incidence in antagonist group ${ }^{(25)}$.

\section{Conclusion:}

The results obtained from this study indicated no statistically significant differences regarding the pregnancy rates or regarding the developing of OHSS complication in the studied population; so larger studies with larger sample size and longer duration is needed to clarify the roles of different IVF protocols.

\section{6-Refernces:}

1-Azziz R, Woods KS, Reyna R et al. (2004): The prevalence and features of the polycystic ovary syndrome in an unselected population. J. Clin. Endocrinol. Metab., 89: 2745-2749.

2-Kousta E, White DM and Franks S (1997): Modern use of clomiphenecitrate in induction of ovulation. Hum. Reprod. Update, 3: 359-365.

3-Rotterdam ESHRE/ASRM-Sponsored PCOS Consensus Workshop Group. (2004): consensus on diagnostic criteria and long-term health risks related to polycystic ovary syndrome. Fertil. Steril., 81: 19-25.

4-Franks S (2002): Adult polycystic ovary syndrome begins in childhood. Best Pract. Res. Clin. Endocrinol. Metab., 16: 263-272

5-Delvigne A and Rozenberg S (2003): Review of clinical course and treatment of ovarian hyperstimulation syndrome (OHSS). Hum. Reprod. Update, 9: 77-96.

6-Homburg R, Berkowitz D, Levy T et al. (1993): In vitro fertilization and embryo transfer for the treatment of infertilty associated with polycystic ovary syndrome. Fertil. Steril., 60: 858-863.

7-Al-Inany HG, Abou-Setta AM and Aboulghar M (2007): Gonadotrophin releasing hormone antagonists for assisted conception: a Cochrane review. Cochrane Database Syst. Rev., 14:640-649

8-Aboulghar MA, Mansour RT, Amin YM et al. (2007): A prospective randomized study comparing coasting with GnRH antagonist administration in patients at risk for severe 
OHSS. Reprod. Biomed Online, 15:271279.

9-Lainas TG, Sfontouris IA, Zorzovilis IZ et al. (2009): Flexible GnRH antagonist protocol versus GnRH agonist long protocol in patients with polycystic ovary syndrome treated for IVF: a prospective randomised controlled trial (RCT). Human Reproduction, 25(3): 683-689.

10-Lee HS, Park HK, Ko JH et al. (2013): Impact of body mass index on luteinizing hormone secretion in gonadotropinreleasing hormone stimulation tests of boys experiencing precocious puberty. Neuroendocrinology, 97(3): 225-231.

11-Bahçeci M, Ulug U, Ben-Shlomo I et al. (2005): Use of a GnRH antagonist in controlled ovarian hyperstimulation for assisted conception in women with polycystic ovary disease: a randomized, prospective, pilot study. The Journal of Reproductive Medicine, 50(2): 84-90.

12-Kaur H, Krishna D, Shetty N et al. (2012): A prospective study of GnRH long agonist versus flexible GnRH antagonist protocol in PCOS: Indian experience. Journal of Human Reproductive Sciences, 5(2): 181186.

13-Ashrafi M, Moini A, Ezabadi Z et al. (2005): A comparative study of $\mathrm{GnRH}$ antagonist and GnRH agonist in PCO patients undergoing IVF/ICSI cycles. Iran J. Reprod. Med., 3:14-18.

14-Huang SY, Huang HY, Yu HT et al. (2011): Low-dose GnRH antagonist protocol is as effective as the long GnRH agonist protocol in unselected patients undergoing in vitro fertilization and embryo transfer. Taiwanese Journal of Obstetrics and Gynecology, 50(4): 432-435.

\section{5-Albano C, Felberbaum $R$ and Smitz $J$} (2000): Ovarian stimulation with HMG: results of a prospective randomized phase III European study comparing the luteinizing hormone-releasing hormone (LHRH)- antagonist cetrorelix and the LHRH-agonist buserelin. Human Reproduction, 15: 526-531.
16-Olivennes F, Cunha-Filho JS, Fanchin R et al. (2002): The use of GnRH antagonists in ovarian stimulation. Hum. Reprod. Update, 2: 8:279-90.

17-Fluker M, Grifo J, Leader A et al. (2001): Efficacy and safety of ganirelixacetateversusleuprolide acetate in women undergoing controlled ovarian hyperstimulation. Fertil. Steril., 75(1): 3845.

18-Orvieto R, Meltcer S, Homburg $\mathrm{R}$ et al. (2009): What is the preferred GnRH analogue for polycystic ovary syndrome patients undergoing controlled ovarian hyperstimulation for in vitro fertilization? Fertility and Sterility, 91(4): 1466-1468.

19-Hosseini MA, Aleyasin A, Saeedi H et al. (2010): Comparison of gonadotropinreleasing hormone agonists and antagonists in assisted reproduction cycles of polycystic ovarian syndrome patients. Journal of Obstetrics and Gynaecology Research, 36(3): 605-610.

20-Kim YJ, Ku SY, Jee BC et al. (2010): A comparative study on the outcomes of in vitro fertilization between women with polycystic ovary syndrome and those with sonographic polycystic ovary-only in GnRH antagonist cycles. Archives of Gynecology and Obstetrics, 282(2): 199205.

21-Lainas TG, Sfontouris IA, Zorzovilis IZ et al. (2010): Flexible GnRH antagonist protocol versus GnRH agonist long protocol in patients with polycystic ovary syndrome treated for IVF: A prospective randomised controlled trial (RCT). Hum. Reprod., 25:683-689.

22-Haydardedeoglu B, Kilicdag E, Parlakgumus A et al. (2012): IVF/ICSI outcomes of the OCP plus GnRH agonist protocol versus the OCP plus GnRH antagonist fixed protocol in women with PCOS: a randomized trial. Archives of Gynecology and Obstetrics, 286(3): 763769.

23-Singh $\mathrm{N}$, Naha $\mathrm{M}$, Malhotra $\mathrm{N}$, et al. (2014): Comparison of gonadotropin- 
releasing hormone agonist with GnRH antagonist in polycystic ovary syndrome patients undergoing in vitro fertilization cycle: Retrospective analysis from a tertiary center and review of literature. Journal of Human Reproductive Sciences, 7(1): 52-56.
24-Mutlu M, Mutlu I, Erdem M et al. (2017): Comparison of the standard GnRH antagonist protocol and the luteal phase estradiol/GnRH antagonist priming protocol in poor ovarian responders. Turkish Journal of Medical Sciences, 47(2): 470-475.

25- Pundir J, Sunkara S, El-Toukhy T et al. (2012): Meta-analysis of GnRH antagonist protocols: do they reduce the risk of OHSS in PCOS?. Reproductive Biomedicine Online, 24(1):6-22. 